\title{
USE OF THE MPS METHOD TO ESTIMATE THE ENERGY CONVERSION EFFICIENCY OF THE OWC-WEC (FIRST REPORT)
}

\author{
Mitsuhiro Masuda* \\ Dept. of Maritime Systems Engineering, \\ Division of Marine Technology, \\ Tokyo University of Marine Science and Technology \\ masuda@kaiyodai.ac.jp \\ Kiyokazu Minami \\ Dept. of Maritime Systems Engineering, \\ Division of Marine Technology, \\ Tokyo University of Marine Science and Technology \\ minami@kaiyodai.ac.jp
}

\author{
Yutaro Sasahara \\ Research Vessel Management Team \\ Metals Mining Technology Department \\ Japan Oil, Gas and Metals National Corporation \\ sasahara-yutaro@jogmec.go.jp \\ Tahsin Tezdogan \\ Dept. of Naval Architecture, \\ Ocean and Marine Engineering, \\ University of Strathclyde \\ tahsin.tezdogan@strath.ac.uk
}

\author{
Atilla Incecik \\ Dept. of Naval Architecture, \\ Ocean and Marine Engineering, \\ University of Strathclyde \\ atilla.incecik@strath.ac.uk
}

\begin{abstract}
In recent years, ocean renewable energy has been increasingly in the spotlight due to global warming and energy issues etc. Research on ocean renewable energy devices is being carried out by many researchers, companies and countries. Additionally, many sea tests are being carried out by countries with real sea test sites. Ocean renewable energy devices still need to research about a power generation method, an energy conversion efficiency and costs etc. In this paper, Authors focus on the oscillating water column type wave energy converter (called below OWC-WEC). It is considered that the OWC-WEC is useful because a movable part which becomes a cause of trouble is few and can also be used as a breakwater device. In this study, the MPS (Moving Particle Semi-implicit) method is applied to the performance analysis of the OWC-WEC. It is because the performance analysis by various parameters and the reduction of an experimental cost is needed. In this paper, these following items are shown; 1) the development of analysis program code which can simulate the multiphase flow by three phases of gas, liquid and solid, 2) the development of air duct model, 3) verification of the usefulness of the improved MPS program code and 4) verification of the applicability to the performance analysis of the OWC-WEC. This program code was applied to the simulation of the fixed type OWC-WEC. Following this, the reproducibility of the primary conversion efficiency of the fixed type OWC-WEC by this program code was verified and discussed. Finally, the usefulness and applicability of this program code were discussed and recommendations for future work were made.
\end{abstract}

Keyword: Ocean Renewable Energy, OWC-WEC, The MPS Method, Primary Conversion Efficiency, Multiphase Flow

\section{INTRODUCTION}

In recent years, ocean renewable energy has been increasingly in the spotlight due to global warming and energy issues etc. For example, in Japan, the demand for ocean renewable energy is growing due to the nuclear power plant accidents of the Tohoku earthquake tsunami disaster on 11th March 2011. Research on ocean renewable energy devices is being conducted by many

\footnotetext{
* Associate Professor and corresponding author.
} 
researchers, companies and countries. Additionally, many sea tests are being conducted by countries with real sea test sites. Many researchers have focused on the oscillating water column type wave energy converter (called below OWC-WEC). It is considered that the OWC-WEC is useful because it has few movable parts and it can also be used as a breakwater device. The OWC-WEC have been thoroughly studied in Japan and it has a long history. [1], [2], [3] However, the OWC-WEC still need further research about energy conversion efficiency and costs etc. Especially, those are significant for Japan where wave energy density is low. Thus, many performance analyses by various parameters are still necessary. Experimental investigations are very expensive to conduct. Therefore, other investigation methods are needed which can conduct tests by various parameters at low cost.

In this study, the MPS (Moving Particle Semi-implicit) method is applied to the performance analysis of the OWC-WEC. The MPS method is a one of a kind particle method in CFD (Computational Fluid Dynamics). The MPS method has the following features; 1) The MPS method is a particle method and it can calculate easily a fully nonlinear phenomenon, 2) It does not involve the making a calculation mesh because it is a meshfree method, 3) The reproduction of the shape of complicated deformations of free water surface is easy and 4) The reproduction of the shape of complicated deformations of free water surface is possible and the reproduction of boundary between air and water is easy. With these features, the MPS method can calculate even the deformation of a water surface in an air chamber of the OWC-WEC, the air pressure and the primary conversion efficiency. There are other CFD methods which can calculate the issues of OWC-WEC. However, it is considered that the calculation method which can calculate the issues of the OWC-WEC including a fully nonlinear phenomenon is limited. In this paper, following items are shown; 1) the development of an analysis program code which can simulate the multiphase flow by three phases of gas, liquid and solid, 2) the development of an air duct model, 3) verification of the usefulness of the improved MPS program code and 4) verification of the applicability to the performance analysis of the OWC-WEC. In previous work, it has been shown about the fundamental applicability of multiphase flow to the floating structure with air chambers called an air-cushion type floating structure. [4] However, there were issues with the pressure vibration of particles and the instability of free water surface in an air chamber in this study. Thus, the present work looks at the high accuracy of the MPS method program code and corrects the multiphase flow algorithm. This program code is applied to the simulation of the fixed type OWC-WEC. Finally, the reproducibility of the primary conversion efficiency of the fixed type OWC-WEC by this program code is verified and discussed.

\section{SIMULATION METHOD}

\subsection{Overview of the MPS Method:}

The MPS Method was first published by Koshizuka and Oka in 1996 [5] and has been used in research in various areas of engineering and science since then. The MPS Method uses the interaction model of particles that corresponds to the differential operator, and the governing equations are discretized by the interaction model. In the discretization, the weight function is applied. The variable of the weight function is the distance between the particles. Since the particles move by retaining such variables as velocity and pressure, they do not require a grid as in the numerical methods of the finite difference method and finite element method etc.

The fluid is assumed to be incompressible and viscous so that the governing equations are the continuity equation and the Navier-Stokes equation as follows;

$$
\begin{gathered}
\nabla \cdot u=\frac{D \rho}{D t}=0 \\
\frac{D u}{D t}=-\frac{1}{\rho} \nabla P+v \nabla^{2} u+g
\end{gathered}
$$

where $u$ is the velocity, $\rho$ is the density of fluid, $t$ is the time, $P$ is the pressure, $v$ is the kinematic viscosity coefficient and $g$ is the gravitational acceleration. Eq. (1) is the continuity equation and indicates that the mass is conserved or vanishes the material derivative of the density. And, it is indicated that the divergence of the velocity is zero. Typically, the formula that the density is constant is used in the MPS method. Eq. (2) is the set of Navier-Stokes equations with the left side being the Lagrange Differential or material derivative. The first term on the right side represents the pressure gradient term, the second term the viscosity term, and finally the third term the gravitational term.

In the MPS method, particle interaction models are prepared for the gradient term and the Laplacian term which are differential operators about spatial directions in the pressure term and the viscosity term. If a particle $i$ has the position vector, and the physical quantity and physical vector, the gradient model, the divergence model and the Laplacian model are represented as follows.

$$
\begin{gathered}
\langle\nabla \phi\rangle_{i}=\frac{d}{n^{0}} \sum_{i \neq j}\left[\frac{\phi_{j}-\phi_{i}}{\left|r_{j}-r_{i}\right|^{2}}\left(r_{j}-r_{i}\right) w\left(\left|r_{j}-r_{i}\right|\right)\right] \\
\langle\nabla \cdot u\rangle_{i}=\frac{d}{n^{0}} \sum_{j \neq i}\left[\frac{\left(u_{j}-u_{i}\right) \cdot\left(r_{j}-r_{i}\right)}{\left|r_{j}-r_{i}\right|^{2}} w\left(\left|r_{j}-r_{i}\right|\right)\right]
\end{gathered}
$$




$$
\left\langle\nabla^{2} \phi\right\rangle_{i}=\frac{2 d}{\lambda n^{0}} \sum_{j \neq i}\left[\left(\phi_{j}-\phi_{i}\right) w\left(\left|r_{j}-r_{i}\right|\right)\right]
$$

where $\phi$ is the physical quantity, $d$ is the number of dimensions, $w$ is the weight function, $n^{0}$ is the initial particle number density and $\lambda$ is the correction coefficient. The weight function is defined as follows.

$$
\mathrm{w}(r)=\left\{\begin{aligned}
\frac{r_{e}}{r}-1 & \left(0 \leq r \leq r_{e}\right) \\
0 & \left(r_{e} \leq r\right)
\end{aligned}\right.
$$

where $r$ is the distance between particles and $r_{e}$ is the influence radius. The particle number density $n_{i}$ is represented as the summation of the weight function with neighbor particles $j$ existing inside the influence radius of the particle $i$.

$$
n_{i}=\sum_{j \neq i} w\left(\left|\vec{r}_{j}-\vec{r}_{i}\right|\right)
$$

In an incompressible fluid, the fluid density is constant. Thus, it is necessary that the particle number density is constant. This constant value which becomes a standard is the initial particle number density $n^{0}$.

In the MPS algorithm, if position $r_{i}^{k}$ and velocity $u_{i}^{k}$ of the particle are known at time $k$, all terms other than the pressure are calculated explicitly and the tentative velocity $u_{i}^{k+1}$ and the position $r_{i}^{k+1}$ can be obtained. The pressure values are obtained by solving the pressure Poisson equation as follows.

$$
\nabla^{2} P^{k+1}=-\frac{\rho_{0}}{\Delta t^{2}} \frac{n^{*}-n^{0}}{n^{0}}
$$

where $\Delta t$ is the time step of the calculation, $n^{*}$ is the particle number density after the calculation is finished explicitly and $\rho_{0}$ is the constant value of the density.

The details of numerical modeling are not described here; for details the reader is referred to the studies given in Koshizuka and Oka [5], [6] and Masuda et al. [7], [8]. However, the multiphase flow model, the improved source term of the pressure Poisson equation, the high accuracy gradient model, the divergence model and the atmospheric pressure model are described below.

\subsection{The High Accuracy Multiphase Flow Model: \\ 2.2.1 The Multiphase Flow Model:}

In this study, it is necessary that the multiphase flow as gas, liquid and solid is considered. The MPS method can be set up with the fluid density of each fluid particle. However, there is a large difference in the densities between air and water. If the density ratio exceeds 10 , the calculation becomes unstable on the boundary between air and water. Thus, the multiphase flow model is applied to this MPS method program code. The multiphase flow model in the MPS method is proposed by Koshizuka et al. [9]

In this algorithm, the calculation of the pressure field in one time step is separated into two steps;

STEP 1: Pressure values of the heavy particles are calculated, where light particles are air particles and heavy particles are particles other than air particles. In this case, when heavy particles lie on the boundary with light particles, it is assumed that they are on the free surface boundary. Normally, the value of zero is given to the pressure value of the free water surface in the MPS method. However, the pressure value on this free surface boundary is not zero and the pressure values of neighboring light particles are given as the Dirichlet boundary condition.

STEP 2: The pressure of light particles is calculated by assuming heavy particles to be wall particles. In that case, the Neumann boundary condition, which is gradient zero, is given to the pressure value of fixed heavy particles.

When calculating the pressure Poisson equation of the light particles, a limited compressibility is considered and the initial particle number density $n^{0}$ in Eq. (8) is improved as follows;

$$
n_{i}^{c}=n^{0}+\frac{n^{0}}{\rho_{0}} \frac{\partial \rho}{\partial P}\left(P_{i}-P_{0}\right)
$$

where $n_{\mathrm{i}}{ }^{0}$ is the corrected initial particle number density of the light particle $i$ and $P_{0}$ is the initial pressure. The limited compressibility can be applied within the range of which the density change can be linearized. It is defined using the speed of sound $c$. 


$$
\frac{\partial \rho}{\partial P}=\frac{1}{c^{2}}
$$

\subsubsection{The Improved Source Term of the Pressure Poisson Equation:}

The MPS method has an issue in that the calculation accuracy is decreased due to the pressure vibration. The cause of the pressure vibration is due to the numerical vibration of the source term of the right side in Eq. (8). In the process of calculation, if the position of particles becomes irregular, it is possible that $n^{*}$ becomes an extremely different value compared with the constant value of the particle number density. Also, the source term of the pressure Poisson equation vibrates spatially.

In this study, the pressure Poisson equation is improved based on Arai et al.'s method [10] as follows;

$$
\left\langle\nabla^{2} P\right\rangle_{i}^{k * 1}=a\left(-\frac{\rho_{i}}{\Delta t^{2}} \frac{n^{*}-n^{0}}{n^{0}}\right)+(1-a) \frac{\rho_{i}}{\Delta t} \nabla \cdot u_{i}^{*}
$$

where $\rho_{i}$ is the density of fluid of the particle $i$ and $u_{i}{ }^{*}$ is the velocity of the particle $i$ after the calculation is finished explicitly. $a$ is the parameter for numerical stability and it is used the value of 0.0 to 1.0. Authors determined the constant $a=0.3$. In Eq. (11), the first term of right side is an incompressible condition about the particle number density. The second term is another incompressible condition of the fluid, and it is indicated the divergence of the velocity is zero. The first term has less accumulation of temporal error. The second term has less accumulation of spatial error. By combining these terms, it is possible to obtain a temporally and spatially smooth pressure distribution.

\subsubsection{The High Accuracy Gradient Model and the High Accuracy Laplacian Model:}

The MPS method has an issue that the preservability of progressive waves is low. Additionally, there is an issue in which the free surface is not stabilized even if the velocity change of the particles is small. [11], [12] There are two factors involved in decreasing the calculation accuracy on the free boundary surface. First, the decrease of the kinetic energy due to the pressure vibration. Second, the accuracy of the gradient model is low. Thus, the high accuracy gradient model is applied in this MPS method program code as follows;

$$
\langle\nabla \phi\rangle_{i}=\left[\frac{1}{n^{0}} \sum_{j \neq i} w\left(\left|r_{j}-r_{i}\right|\right) \frac{\left(r_{j}-r_{i}\right)}{\left|r_{j}-r_{i}\right|} \otimes \frac{\left(r_{j}-r_{i}\right)}{\left|r_{j}-r_{i}\right|}\right]^{-1} \cdot\left[\frac{1}{n^{0}} \sum_{j \neq i} w\left(\left|r_{j}-r_{i}\right|\right) \frac{\left(\phi_{j}-\phi_{i}\right)}{\left|r_{j}-r_{i}\right|} \frac{\left(r_{j}-r_{i}\right)}{\left|r_{j}-r_{i}\right|}\right]
$$

where $\otimes$ is the tensor product. This model was applied by Iribe and Nakaza [13] to the analysis of incompressible fluid. By this Equation, the calculation accuracy around a boundary is maintained and the calculation accuracy is improved more than the previous method. The high accuracy divergence model is applied as shown in Eq. (13). In these models, the primary convergent is ensured by Tamai et al. [14]

$$
\langle\nabla \cdot \mathrm{T}\rangle_{i}=\left[\sum_{j \neq i} w\left(\left|r_{j}-r_{i}\right|\right) \frac{\left(r_{j}-r_{i}\right)}{\left|r_{j}-r_{i}\right|} \otimes \frac{\left(r_{j}-r_{i}\right)}{\left|r_{j}-r_{i}\right|}\right]^{-1} \cdot\left[\sum_{j \neq i} w\left(\left|r_{j}-r_{i}\right|\right) \frac{\left(r_{j}-r_{i}\right)}{\left|r_{j}-r_{i}\right|} \otimes \frac{\left(\mathrm{T}_{j}-\mathrm{T}_{i}\right)}{\left|r_{j}-r_{i}\right|}\right]
$$

where $\mathrm{T}$ is the tensor. Normally, a weighted average in the MPS method is calculated by using the number of dimensions $d$ and the initial particle number density $n^{0}$. In these models, the weighted average is calculated based on the relative position of total neighboring particles calculated on the inverse matrix of the right side of Eq. (12) and Eq. (13). By using these models, it is possible to calculate the gradient and the divergence with high accuracy.

\subsubsection{The High Accuracy Atmospheric Pressure Model:}

In the case of using the multiphase flow model in Section 2.2.1, the action direction is not considered because only the pressure value of neighboring air particles is given to the free surface boundary. Thus, the existence effect of the air particles is not considered to the displacement of the free surface boundary. Consequently, the shape of the free surface boundary becomes unstable. A standard atmospheric pressure model is derived by Shibata et al. [15] In this study, the atmospheric pressure model for high accuracy multiphase flow calculation is applied. The pressure Poisson equation considering the atmospheric pressure is shown as follows;

$$
\frac{2 d}{\rho_{i, j} \lambda n^{0}} \sum_{\substack{j \neq i \\ \text { type }(j) \neq \text { gas }}}\left[\left(P_{j}^{k+1}-P_{i}^{k+1}\right) w\left(\left|r_{j}-r_{i}\right|\right)\right]+\frac{2 d}{\rho_{i, j} \lambda n^{0}} \sum_{\substack{j \neq i \\ \text { type }(j)=g a s}}\left[\left(P_{j}^{g a s}-P_{i}^{k+1}\right) w\left(\left|r_{j}-r_{i}\right|\right)\right]
$$




$$
=-(1-\alpha) \frac{1}{\Delta t} \nabla \cdot u^{*}-\alpha \frac{1}{\Delta t^{2}} \frac{\left(n_{i}^{*}-n^{0}\right)}{n^{0}}
$$

In this multiphase flow calculation, the gas particles are existing outside the free surface boundary. Thus, the summation of pressure is calculated directly by the interaction between the liquid particles and the gas particles on the free surface boundary. Here, $P^{\text {gas }}$ must already be known. The pressure value from the previous time step is applied to $P^{g a s}$. From Eq. (14), the simultaneous linear equations of pressure are obtained as follows;

$$
\begin{aligned}
& \frac{2 d}{\rho_{i, j} \lambda n^{0}} \sum_{\substack{j \neq i \\
t y p e(j) \neq g a s}}\left[\left(P_{j}^{k+1}-P_{i}^{k+1}\right) w\left(\left|r_{j}-r_{i}\right|\right)\right]+\frac{2 d}{\rho_{i, j} \lambda n^{0}} \sum_{\substack{j \neq i \\
\text { type }(j)=g a s}}\left[\left(P_{j}^{g a s}-P_{i}^{k+1}\right) w\left(\left|r_{j}-r_{i}\right|\right)\right] \\
& =-\frac{2 d}{\rho_{i, j} \lambda n^{0}} \sum_{\substack{j \neq i \\
\text { type }(j)=\text { gas }}}\left[\left(P_{j}^{g a s}\right) w\left(\left|r_{j}-r_{i}\right|\right)\right]-\left\{(1-\alpha) \frac{1}{\Delta t} \nabla \cdot u^{*}-\alpha \frac{1}{\Delta t^{2}} \frac{\left(n_{i}^{*}-n^{0}\right)}{n^{0}}\right\}
\end{aligned}
$$

If the particle $i$ is existing in the free water surface or if the free water surface particles are existing in the influence radius of the particle $i$, Eq. (14) is applied. In addition, the particle number density must be $n_{i}{ }^{*}<n^{0}$.

Then, the gradient model considering the atmospheric pressure is shown as follows;

$$
\begin{aligned}
\langle\nabla P\rangle_{i} & =\left[\sum_{\substack{j \neq i \\
t y p e \\
(j) \neq g a s}} w\left(r_{j}-r_{i}\right) \frac{\left(r_{j}-r_{i}\right)}{\left|r_{j}-r_{i}\right|} \otimes \frac{\left(r_{j}-r_{i}\right)}{\left|r_{j}-r_{i}\right|}\right]^{-1}\left(\frac{1}{n^{0}} \sum_{\substack{j \neq i \\
t y p e(j) \neq g a s}}\left[\left(P_{j}-\widehat{P}_{l}\right) \frac{\left(r_{j}-r_{i}\right)}{\left|r_{j}-r_{i}\right|^{2}}\right]\right) \\
& +\left[\sum_{\substack{j \neq i \\
\text { type }(j) \neq g a s}} w\left(r_{j}-r_{i}\right) \frac{\left(r_{j}-r_{i}\right)}{\left|r_{j}-r_{i}\right|} \otimes \frac{\left(r_{j}-r_{i}\right)}{\left|r_{j}-r_{i}\right|}\right]^{-1}\left(\frac{1}{n^{0}} \sum_{\substack{j \neq i \\
\text { type }(j) \neq g a s}}\left[\left(P_{j}-\widehat{P}_{l}\right) \frac{\left(r_{j}-r_{i}\right)}{\left|r_{j}-r_{i}\right|^{2}}\right]\right)
\end{aligned}
$$

\subsection{Reproduction of an Air Chamber:}

\subsubsection{Nozzle boundary Conditions and Judgement Conditions of the air particles:}

The multiphase flow model described earlier has some issues. Firstly, the air in the air chamber is reproduced by gas particles. If inflow and outflow of the air are considered at the nozzle in the air chamber, it is necessary that the gas phase outside the air chamber is considered. This means that the calculation cost is increased. Secondly, if the free water surface in the air chamber goes down, the pressure value of upper air particles decreases, and it is judged that there lies the free surface boundary. For that reason, non-physical blank areas occur in the air chamber. Finally, the pressure value below the initial pressure value becomes zero. Thus, it is possible that the pressure values in the air chamber are not sufficiently reproduced. In this study, the nozzle boundary model is applied. Then, the judgement condition of the free surface is improved and the application of the initial pressure value to the gas particles is abolished.

The nozzle boundary conditions are developed based on the transparent conditions by Shibata et al. [16] The schematic diagram of the nozzle boundary is shown in Figure 1. The nozzle boundary is installed on the top of the air chamber. There are two cells (cells A and B) on the inside of the nozzle boundary as shown in Figure 1. Each cell is a square with length of side $l_{0}$. The particles in cells A and B are moving at a specific velocity. When the pressure conditions are given, it is necessary that the pressure drop when passing through the nozzle is considered. If the atmospheric pressure (which is the initial pressure for calculation) is $P_{0}$ and the pressure which is given as the Dirichlet boundary conditions to the nozzle boundary is $P_{N}$, both relationships are shown by Eq. (17) using the pressure drop on the nozzle $\Delta P$.

$$
\begin{gathered}
P_{0}=P_{N}+\Delta P_{N} \\
\Delta P_{N}=C_{p} \cdot \frac{1}{2} \rho_{a} \cdot v_{N}^{2} \cdot \operatorname{sign}\left(v_{N}\right)
\end{gathered}
$$

where $C_{p}$ is the pressure drop coefficient on the nozzle, $\rho_{a}$ is the air density and $v_{N}$ is the vertical flow velocity on the nozzle. The pressure drop coefficient is calculated by Eq. (19).

$$
C_{p}=\left(\frac{1}{C_{d} \varepsilon}\right)^{2}, \varepsilon=\frac{A_{N}}{A_{W}}
$$


where $A_{W}$ is the area of the air chamber, $A_{N}$ is the cross section area of the nozzle and $\varepsilon$ is the nozzle ratio.

Thus, the pressure which is given as the Dirichlet boundary conditions to the nozzle boundary is shown as follow.

$$
P_{N}=P_{0}-C_{p} \cdot \frac{1}{2} \rho_{a} \cdot v_{N}^{2} \cdot \operatorname{sign}\left(v_{N}\right)
$$

where $v_{N}$ is the arithmetic mean of the air particles contained in cells A and B.

When the pressure function with the flow velocity as an argument is given to the nozzle boundary, a pressure difference occurs depending on the fluctuation of the mass in between the nozzle boundary section and the air chamber section. If the pressure value in the air chamber section is higher than the nozzle boundary section, the air particles go out from the nozzle boundary. In this case, if the air particles pass the nozzle boundary, those air particles are excepted from calculation. On the other hand, if the pressure value in the air chamber section is lower than the nozzle boundary section, the air particles flow from the outside to the inside through the nozzle boundary. In this time, if no air particle exists in the A cell due to the inflow, one air particle is generated in that cell. This air particle is generated so that the distance of the generating air particle and the air particles in the $\mathrm{B}$ cell is near the length of the A cell $l_{0}$. The air particles through the nozzle boundary are imitated as a uniform flow. Thus, the form of the nozzle boundary does not change. The nozzle boundary is excepted from the pressure calculation together with the wall particles.

In this study, the nozzle boundary is considered as the orifice. If turbine characteristics of the different pressure coefficients, the torque coefficient and the flow coefficient etc. are known, each turbine influence can be reproduced by the pressure drop model based on each characteristic parameter.

Then, the judgement condition of the free surface and the treatment of the pressure value below the initial pressure value are corrected for the air particles in the air chamber. Normally, the pressure value below the initial pressure value becomes zero. This means that there are particles on the free surface. In this case, the gravitational force between particles by the pressure gradient does not work. For that reason, the free surface is calculated, and the non-physical blank areas occur in the air chamber.

Thus, the judgement of the free surface is not performed other than the particles neighboring the nozzle boundary. However, the particles neighboring the nozzle boundary is given the Dirichlet boundary conditions. As the result, the judgement condition of the free surface is not applied to the neighboring the nozzle boundary and the judgement of the free surface is excepted to all air particles in the air chamber. Then, the pressure value zero is not applied to the air particles. This means that the negative pressure is considered. If the negative pressure is considered, the gravitational force occurs between particles and the non-physical blank areas are not occur. These improvements are not applied other than the air particles.

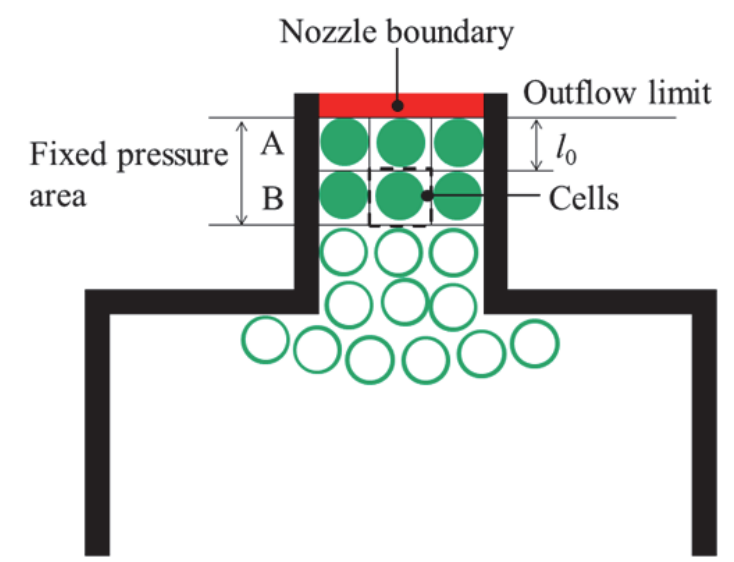

Figure 1 Schematic diagram of the nozzle boundary

\subsubsection{Confirmation of the reproducibility of the Air Chamber: \\ (1) Calculation Conditions:}

The reproducibility of the air chamber is confirmed by comparisons of the calculation results of the normal MPS method and the improved MPS method. A two dimensional numerical test is performed. The calculation setup system is shown in Figure 2. The air chamber, which is a square of $0.4 \mathrm{~m}$ sides, is set as the main part of the calculation area as shown in Figure 2. In the bottom of the air chamber, an oscillating wall boundary is installed. This imitates the free water surface. In the case of the normal MPS method program code (here on termed, the previous model), the air tank on the duct is installed. because the previous model had no nozzle boundary. Thus, the air tank is needed for the simulation of the inflow and outflow of the air. The height of the duct is $0.15 \mathrm{~m}$. The height of the tank is $1.6 \mathrm{~m}$ and the width of the tank is $0.8 \mathrm{~m}$. In the case of the improved MPS method (called below, present method), the nozzle boundary is set $0.05 \mathrm{~m}$ above the ceiling of the air chamber. In both cases, the width of the duct is $0.02 \mathrm{~m}$. The calculation conditions are shown in Table 1. In the case of the previous model, the total number of particles is 25,286 . In the case of the present model, the total number of particles is 3,604 . The distance between particles is $0.008 \mathrm{~m}$. The 
amplitude of the oscillating wall boundary is $0.07 \mathrm{~m}$ and the period is $1.15 \mathrm{sec}$. Regarding both models, the snapshots of calculation results are compared. In addition, the air pressures and the air flow velocities are compared.

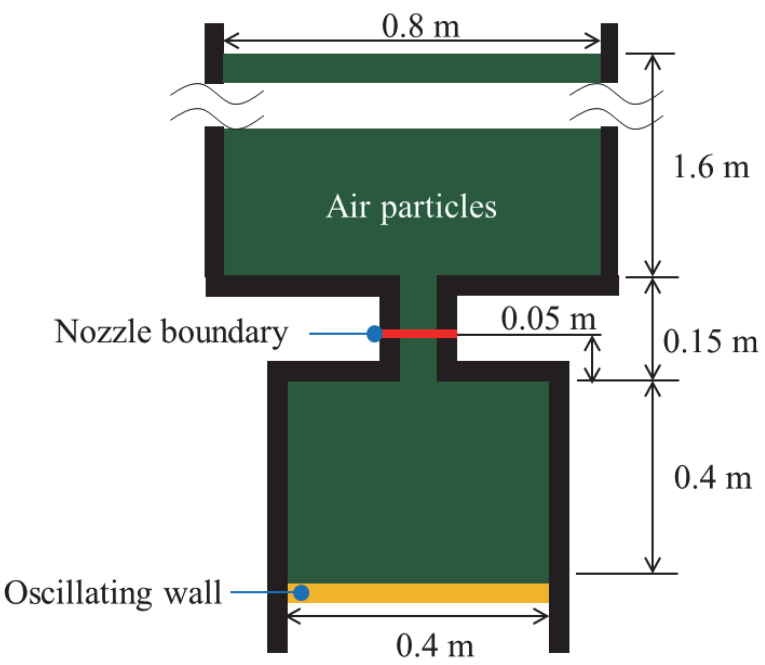

Figure 2 Numerical air chamber for the forced oscillation problem

Table 1 Calculation conditions for the air chamber analysis

\begin{tabular}{l|l}
\hline \hline Item & Value \\
\hline \hline Number of particles (Present model) $[-]$ & 3,604 \\
Number of particles (Previous model) [-] & 25,286 \\
Distance between particles [m] & 0.008 \\
Simulation time $[\mathrm{s}]$ & 10.0 \\
Density of particles $\left[\mathrm{kg} / \mathrm{m}^{3}\right]$ water / air & $999.1 / 1.226$ \\
\hline Oscillating amplitude $[\mathrm{m}] /$ period $[\mathrm{s}]$ & $0.07 / 1.15$ \\
\hline Reference Pressure at nozzle $[\mathrm{Pa}]$ & 20.0 \\
\hline
\end{tabular}

\section{(2) Results and discussions:}

The results are presented below. The comparison of air pressures at the ceiling of the air chamber is shown in Figure 3. The comparison of air flow velocities at the ceiling of the air chamber is shown in Figure 4. Snapshots of the air flow velocity by the previous model are shown in Figure 5. Snapshots of the air flow velocity by the present model are shown in Figure 6.

First, considering the total number of particles, the calculation cost of the present model is $1 / 7$ th that of the previous model shown in Table 1. The total calculation time of the previous model is $41,243 \mathrm{sec}$. and the total calculation time of the present model is 3,311 sec. Comparing with these calculation times, the calculation cost of the present model is $1 / 12$ th that of the previous model. It is confirmed that the nozzle boundary is effective for the calculation cost reduction.

Next, in the case of the previous model, it is confirmed that air pressure values less than zero cannot be calculated as shown in Figure 3. In the case of air pressure values greater than zero, the pressure vibration is large like a collision force. When the oscillating wall boundary goes down, it is confirmed that the non-physical blank area occurred as shown in Figure 5. These problems are the influence of the correcting condition of pressure values below zero and the free surface boundary condition to air particles. Thus, the air pressure at the ceiling of the air chamber by the previous model is similar to the collision force between the air particles and the wall particles as shown in Figure 3. In the case of the present model, it is confirmed that an air pressure less than zero can be calculated as shown in Figure 3. It is also confirmed that the free surface boundary and the non-physical blank areas do not occur as shown in Figure 6. This is the influence of the negative pressure and the gravitational force between particles by the improved judgement conditions of the air particles. This wave shape is similar to a triangle wave. This is the wave shape where the pressure boundary condition at the nozzle part is two times that of the flow velocity as shown in Eq. (18) and based on that flow direction. In that case, the wave shape of the air pressure does not become a smooth linear wave shape. In the case of the previous model, when air particles flow into the air chamber, it is confirmed that the air flow velocity becomes constant as shown in Figure 4. This problem is the influences of the correcting condition of pressure values below zero and the free surface boundary condition to air particles. When air particles flow into the air chamber, the inflowing air particles are not affected by the air particles in the air chamber because the free surface boundary and the non-physical blank areas occur in the air chamber. However, it is confirmed that the present model can calculate the interaction between the inflow and outflow in the air chamber as shown in Figure 4 and Figure 6. From these results, it is confirmed that the present model can calculate the phenomenon of the inflow and outflow in the air chamber. 


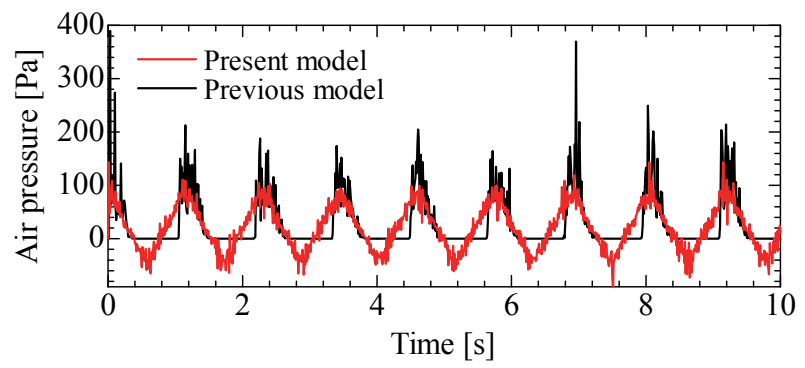

Figure 3 Comparison of air pressures at the ceiling of the air chamber

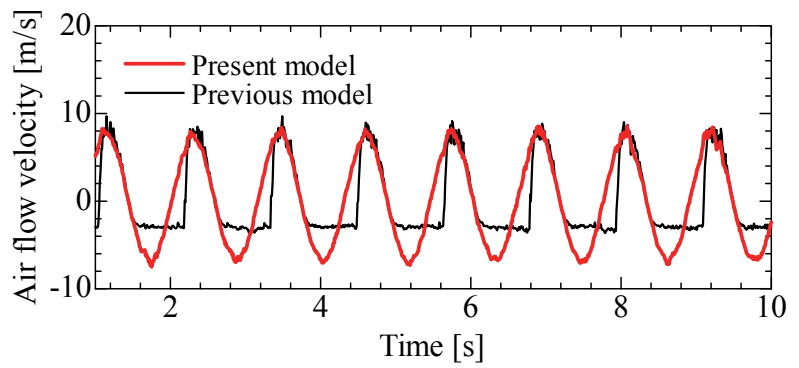

Figure 4 Comparison of air flow velocities at the ceiling of the air chamber

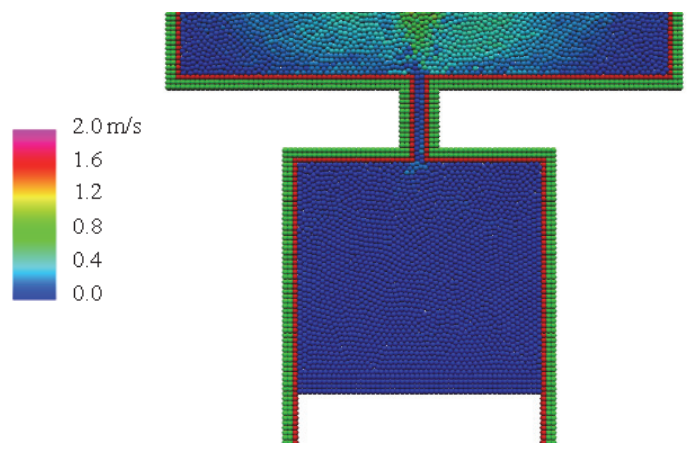

a) $2.59 \mathrm{sec}$.

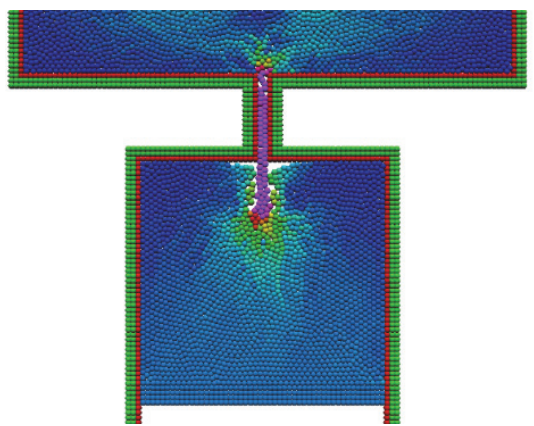

b) $2.75 \mathrm{sec}$.

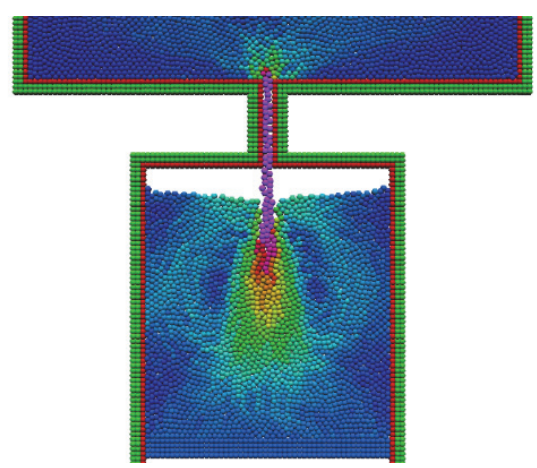

c) $3.05 \mathrm{sec}$.

Figure 5 Snapshots of calculation result by the previous model

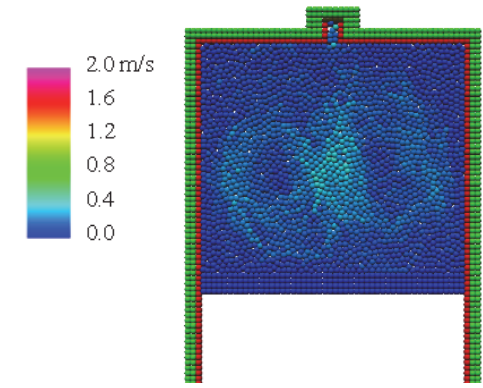

d) $2.59 \mathrm{sec}$.

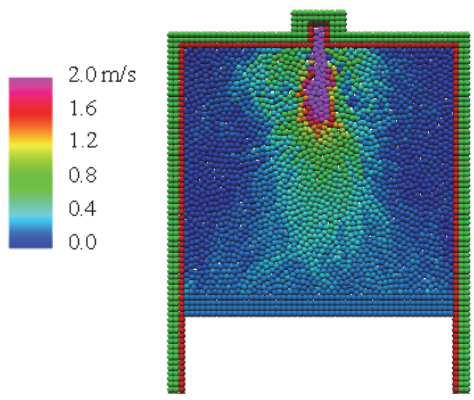

e) $2.75 \mathrm{sec}$.

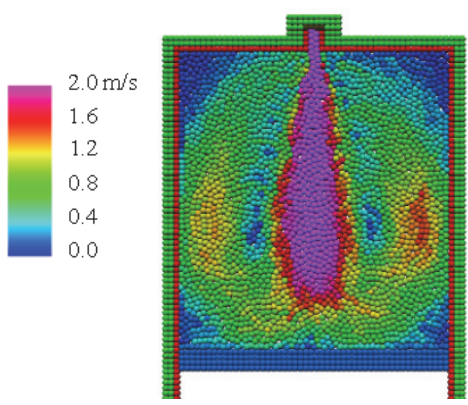

f) $3.05 \mathrm{sec}$.

Figure 6 Snapshots of calculation result by the present model 


\section{PERFORMANCE ANALYSIS OF OWC TYPE WAVE ENERGY CONVERTER}

\subsection{Calculation Conditions:}

In this section, a two dimensional numerical simulation is conducted for simulating the experimental results of the fixed type OWC-WEC by Kojima et al. [17] The calculation setup system is shown in Figure 7. The water depth is $0.6 \mathrm{~m}$. The length of the water tank is 2 times that of the incident wave length $\lambda$. The piston type wave maker boundary is set on the left hand side. This wave maker boundary is an absorbing wave maker and the theories of the non-reflecting type wave makers are applied. [18] The length of the air chamber is $0.4 \mathrm{~m}$. The height of the air chamber is $0.4 \mathrm{~m}$ and the depth of the curtain wall on the front of the air chamber is $0.2 \mathrm{~m}$. In the experimental setup, the thickness of the curtain wall is $0.02 \mathrm{~m}$. In this simulation, the thickness of the curtain wall is $0.05 \mathrm{~m}$ because the air chamber is configured by the wall particles. If the thickness of the curtain wall is less than the influence radius, particles on both sides of the curtain wall will interact. In this study, the influence radius is 3.1 times the distance between particles $l_{0}$. The nozzle, which is $0.11 \mathrm{~m}$ of the length and $0.048 \mathrm{~m}$ of the width is set on the center of the ceiling of the air chamber. This width of the nozzle is different to the experimental setup by Kojima et al. because the width of the nozzle as more than six particles is needed for keeping the calculation accuracy. The pressure drop coefficient given to the nozzle boundary is adjusted for the influence of the difference in the width of the nozzle. The nozzle ratio is the ratio with the water plane area in the air chamber and the cross-sectional area of the nozzle. Regarding the incident wave conditions, the wave heights used are $0.05 \mathrm{~m}, 0.1 \mathrm{~m}, 0.15 \mathrm{~m}$ and $0.20 \mathrm{~m}$, and the wave periods used are as $1.15 \mathrm{sec}, 1.5 \mathrm{sec}$ and $2.0 \mathrm{sec}$. In this section, the wave periods: $1.15 \mathrm{sec}, 1.5 \mathrm{sec}$ and $2.0 \mathrm{sec}$ are called as Case 1, Case 2 and Case 3 from left to right. The wave heights: $0.05 \mathrm{~m}, 0.1 \mathrm{~m}$, $0.15 \mathrm{~m}$ and $0.20 \mathrm{~m}$ are termed 1, 2, 3 and 4, respectively. For example, the case of the wave height: $0.05 \mathrm{~m}$ and the wave period: $1.15 \mathrm{sec}$ is shown as Case 1-1.

Calculation conditions are shown in Table 2. The distance between particles is $0.008 \mathrm{~m}$. The size of the calculation area is decided by the wave period. The initial particle numbers for each calculation area are Case 1: 52,249 numbers, Case 2: 74,299 numbers and Case 3: 104,467 numbers. The simulation times are Case 1: $12.65 \mathrm{sec}$, Case 2: $17.6 \mathrm{sec}$ and Case 3: $22.0 \mathrm{sec}$. The measuring points in the air chamber are shown in Figure 8. Analytical items are the pressure and the water elevation in the air chamber and the primary conversion efficiency. The pressure value is the average value of the pressures measured at four points in the ceiling of the air chamber as shown in Figure 8. The water elevation is measured at three points on the free water surface in the air chamber as shown in Figure 8. The primary conversion efficiency is represented by the pneumatic power $E_{e}$ which is the wave energy absorbing by the air chamber at a unit of time and the power $E_{i}$ of the incident wave. Here, the power $E_{i}$ of the incident wave is represented by the small amplitude wave theory:

$$
E_{i}=\frac{1}{2} \rho g a^{2} c_{g}
$$

where, $\rho$ is the fluid density, $g$ is the gravitational acceleration, $a$ is the incident wave amplitude and $c_{g}$ is the group velocity.

The pneumatic power $E_{e}$ is calculated by the water area $A_{w}$ in the air chamber, and the pressure $P_{(t)}$ and the water elevation $\eta_{(t)}$ in the air chamber for one period of the incident wave as shown in Eq. (22).

$$
\begin{aligned}
E_{e} & =\frac{A_{w}}{T} \int_{0}^{T} P(t) \frac{\partial \eta(t)}{\partial t} d t \\
& =\frac{A_{w}}{T} \sum_{i=1}^{N} P_{i} \cdot \frac{\eta_{i+1}-\eta_{i}}{\Delta t} \Delta t \\
& =\frac{A_{w}}{T} \sum_{i=1}^{N} P_{i}\left(\eta_{i+1}-\eta_{i}\right)
\end{aligned}
$$

From Eq. (21) and Eq. (22), the primary conversion efficiency is represented as Eq. (23).

$$
E=\frac{E_{e}}{E_{i}}
$$




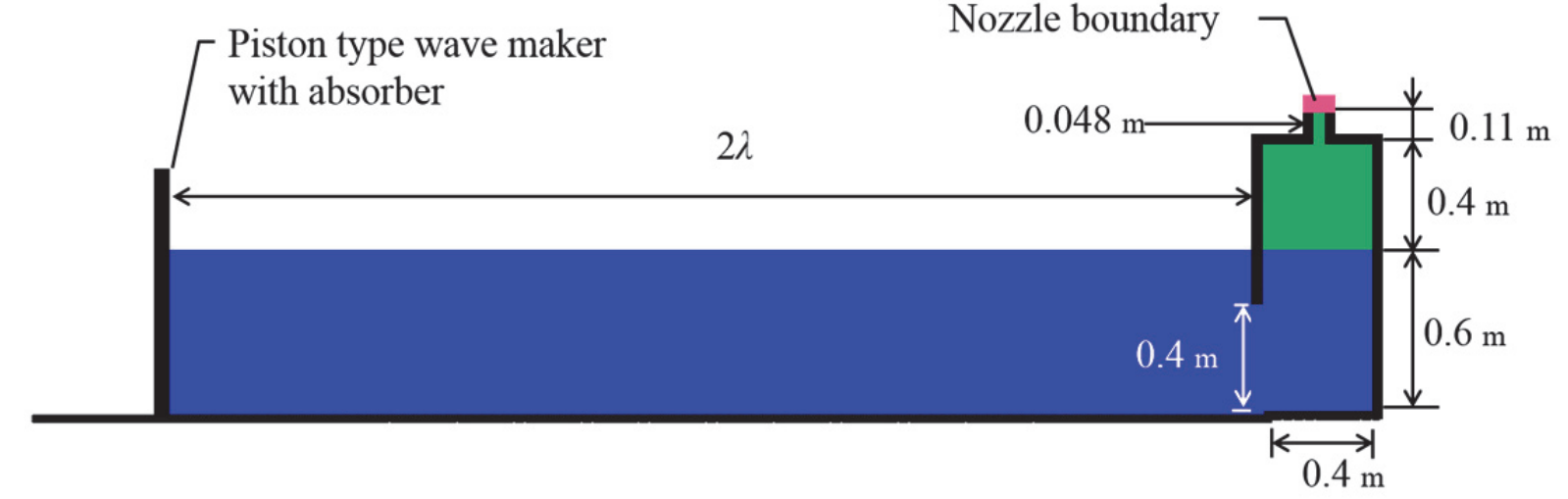

Figure 7 Numerical water tank for performance analysis of the fixed type OWC-WEC

Table 2 Calculation conditions for performance analysis of the fixed type OWC-WEC

\begin{tabular}{|c|c|c|c|}
\hline \multirow{2}{*}{ Item } & \multicolumn{3}{|c|}{ Value } \\
\hline & Case 1 & Case 2 & Case 3 \\
\hline Initial number of particles [-] & 52,249 & 72,499 & 104,467 \\
\hline Distance between particles [m] & & 0.008 & \\
\hline Simulation time $[\mathrm{s}]$ & 12.65 & 17.60 & 22.0 \\
\hline Time step $[\mathrm{s}]$ & & $2.5 \times 10^{-4}$ & \\
\hline Kinematic viscosity $\left[\mathrm{m}^{2} / \mathrm{s}\right]$ & & $1.14 \times 10^{-6}$ & \\
\hline Kinematic viscosity $\left[\mathrm{m}^{2} / \mathrm{s}\right]$ & & $1.47 \times 10^{-5}$ & \\
\hline Density of liquid $\left[\mathrm{kg} / \mathrm{m}^{3}\right]$ & & 999.099 & \\
\hline Density of gas $\left[\mathrm{kg} / \mathrm{m}^{3}\right]$ & & 1.226 & \\
\hline Incident wave period [s] & 1.15 & 1.50 & 2.0 \\
\hline Incident wave height [m] & & $0.05,0.10,0.15,0.20$ & \\
\hline
\end{tabular}

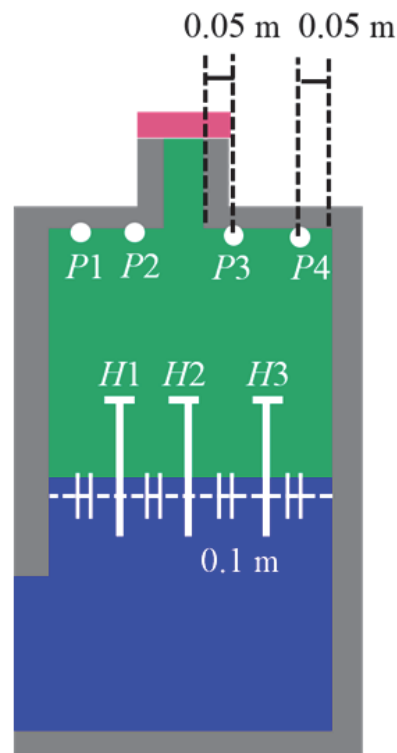

Figure 8 Measuring points in the air chamber

\subsection{Results and Discussion:}

The result from this work are discussed in the following section. Time histories of the water elevation around and inside the air chamber of Case 1 to 4 are shown in Figure $9 . \mathrm{H}_{\mathrm{Syn}}$ is the water elevation of the front of the air chamber. $\mathrm{H}_{\text {Syn }}$ is measured at $0.02 \mathrm{~m}$ front of the air chamber. $\mathrm{H} 1$ to $\mathrm{H} 3$ are the water elevation in the air chamber shown in Figure 8 . Time histories of the air chamber and the water elevation velocity in the air chamber of Case 1 to 4 are shown in Figure 10. This pressure value is the average value of $\mathrm{P} 1$ to $\mathrm{P} 4$ shown in Figure 8. Snapshots of the calculation results of Case 1-4 are shown in Figure 11. In Figure 9 and Figure 10, the broken lines (a), (b) and (c) show each time step in Figure 11. In the case of the broken line (a), the water 
elevation in the air chamber is zero as shown in Figure 11(a). In the case of the broken line (b), the water elevation in the air chamber is the maximum in this time history. In the case of the broken line (c), the water elevation in the air chamber is the minimum in this time history. The comparison of the non-dimensional water elevations is shown in Figure 12. The comparison of the non-dimensional positive pressures is shown in Figure 13. The comparison of the non-dimensional negative pressures is shown in Figure 14. The comparison of the primary conversion efficiency is shown in Figure 15. In Figures 12 to 15 , those simulation results are compared with the experimental values from Kojima et al. [17] In Figure 12, the vertical axis is the ratio of the total amplitude $\eta$ of the water elevation in the air chamber to the incident wave height $H$. In Figures 13 to 15 , the horizontal axis is the ratio of the water depth $h$ to the wave length $L$.

From Figure 9, it can be seen that there is a phase difference between the water elevation of the front of the air chamber and the water elevation in the air chamber. The incident wave passes through the curtain wall section to the inside of the air chamber. It can be considered that this phase difference is the influence due to the curtain wall. From comparison between $\mathrm{H}_{\mathrm{Syn}}$ and other lines, the water elevations in the air chamber are smaller than $\mathrm{H}_{\text {Syn }}$. The pressure from air is small. However, a nozzle is in the air chamber. The water elevation in the air chamber becomes small by the reaction force from the nozzle. There is a load spring due to the nozzle reaction force to the water column. From comparison of Lines H1 to H3, it can be confirmed that there is a difference in the water elevations in the air chamber. It can be considered that the difference between $\mathrm{H} 1$ and $\mathrm{H} 2$ is influenced from the vortex occurring around the tip of the curtain wall. The incident wave flows into the air chamber while making the vortex around the tip of the curtain wall. With this effect, it is considered that the water elevation close to the tip of the curtain wall becomes smaller than the opposite side in the air chamber as shown in Figure 11 (b). From Figure 10, it can be confirmed that the phase of the pressure and the water elevation velocity is similar. It can also be confirmed that the phase of the water elevation $\mathrm{H}_{\text {Syn }}$ the pressure and the velocity is similar as shown in Figure 9 and Figure 10. In addition, when the water elevation becomes a maximum or minimum value, the pressure value becomes zero. If the water elevation reaches a maximum or minimum value, the air pressure affecting the air chamber becomes zero because the rate of change of the volume in the air chamber becomes zero. On the other hand, the non-physical blank areas do not occur as shown in Figure 11. Therefore, this high accuracy multi-phase MPS program can calculate these phenomena with good accuracy.

From Figures 12 to 15 , it can be confirmed that the simulation results and the experimental results are broadly similar. When the incident wave with high energy flows into the air chamber, the amplitude of the water surface becomes low by the load spring due to the nozzle reaction force. In that case, the pressure value becomes high. It can be confirmed that the present method can broadly reproduce that phenomenon as shown in Figures 12 to 14. However, the dispersion of the pressure values is large as shown in Figure 13 and Figure 14. In particular, when the incident wave height is low, that tendency becomes strong. This cause is considered to be due to the three-dimensional effects at the nozzle and the influence of the width of the curtain wall. A two-dimensional simulation is conducted in this study. Thus, these simulation results do not sufficiently consider the pressure drop due to three-dimensional effects. In this case, it is considered that the error between the simulation results and the experimental results becomes small by using the pressure drop coefficient considering three-dimensional effects. On the other hand, the curtain wall in this simulation is two times thicker than the experimental model. Around the curtain wall, a vortex occurs. If the depth of the curtain wall becomes large, the influences of the vortex are seen. [19] Therefore, it is considered that the energy loss around the curtain wall in the simulation results becomes large compared with the experimental results. In order to improve the calculation accuracy, it is necessary to increase the spatial resolution. In the MPS method, this can be done by increasing the number of particles and calculation time. Thus, it is necessary to increase the spatial resolution of the limited part such as the wall of the air chamber. The primary conversion efficiency is broadly similar between the simulation values and the experimental values as shown in Figure 15. However, when the wave height is low, the calculation accuracy becomes low due to the three-dimensional effects and the influences of the depth of the curtain wall. In the next step, it is necessary that these issues are solved and the water tank experiment for these simulation results is conducted.

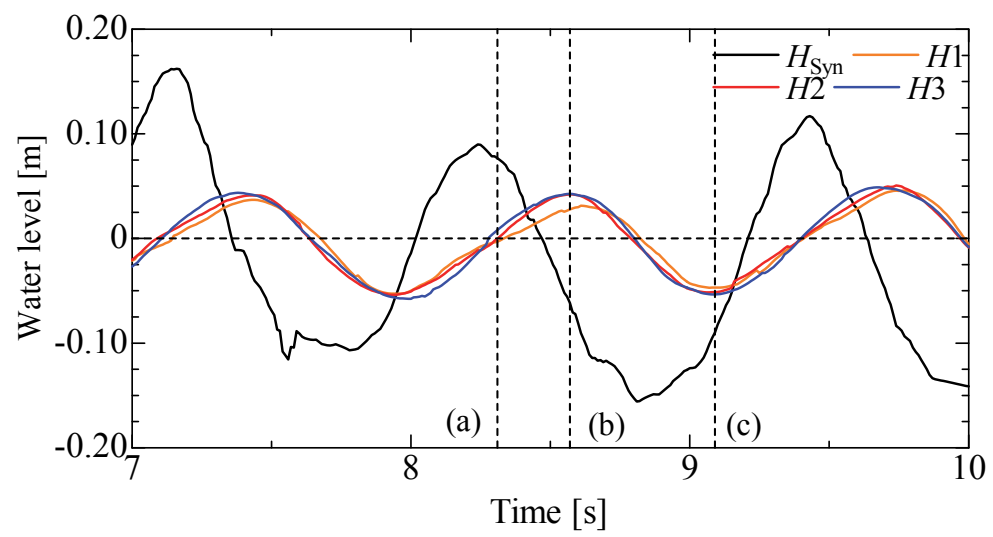

Figure 9 Time histories of the water elevation of the front and the inside of the air chamber of Case $1-4(\mathrm{~T}=1.15 \mathrm{sec}, \mathrm{H}=0.20 \mathrm{~m})$ 


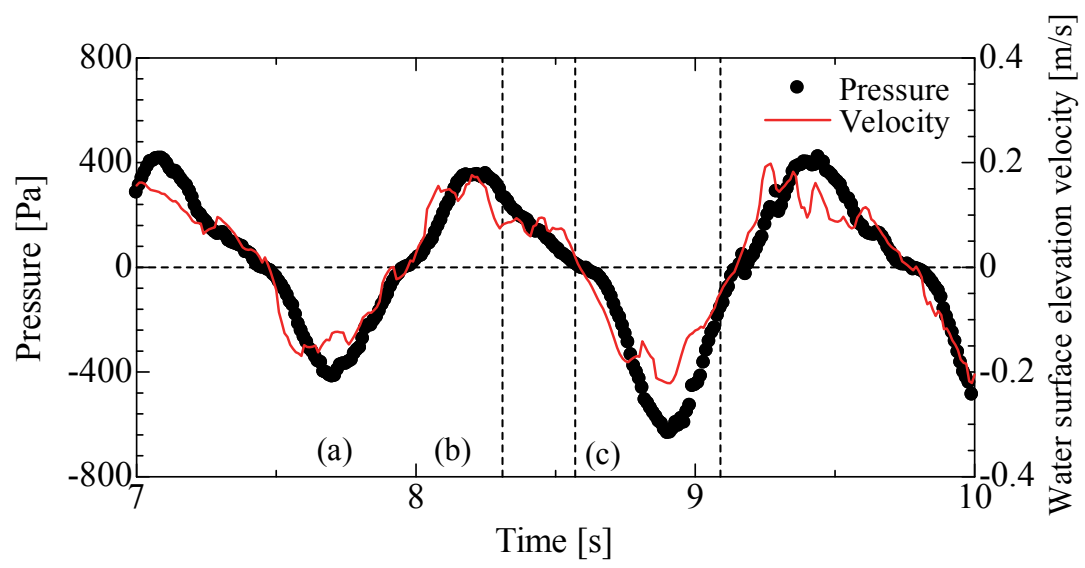

Figure 10 Time histories of the air pressure and the water elevation velocity in the air chamber of Case 1-4 $(\mathrm{T}=1.15 \mathrm{sec}, \mathrm{H}=0.20 \mathrm{~m})$

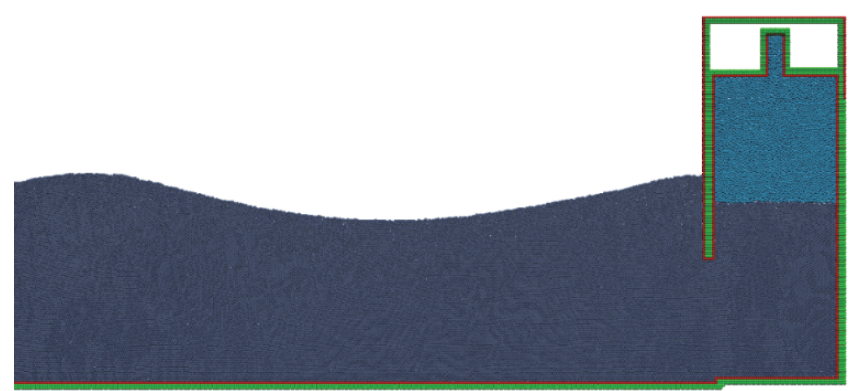

(a) $8.31 \mathrm{sec}$

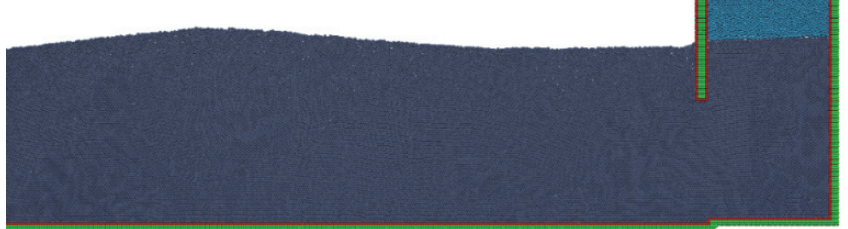

(b) $8.57 \mathrm{sec}$

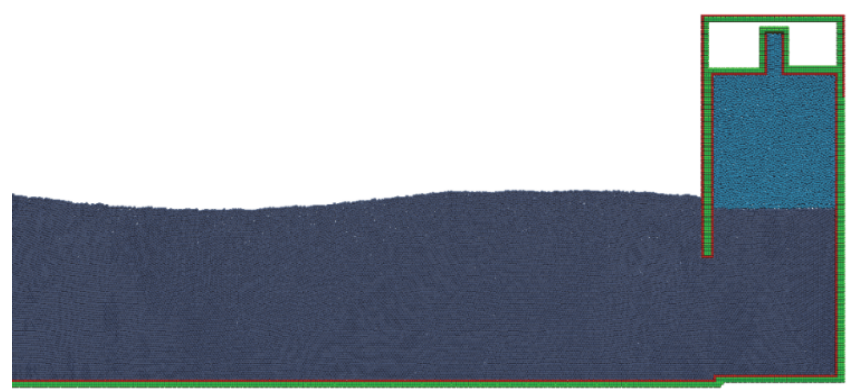

(c) $9.09 \mathrm{sec}$

Figure 11 Snapshots of the simulation result of Case 1-4 $(\mathrm{T}=1.15 \mathrm{sec}, \mathrm{H}=0.20 \mathrm{~m})$ 


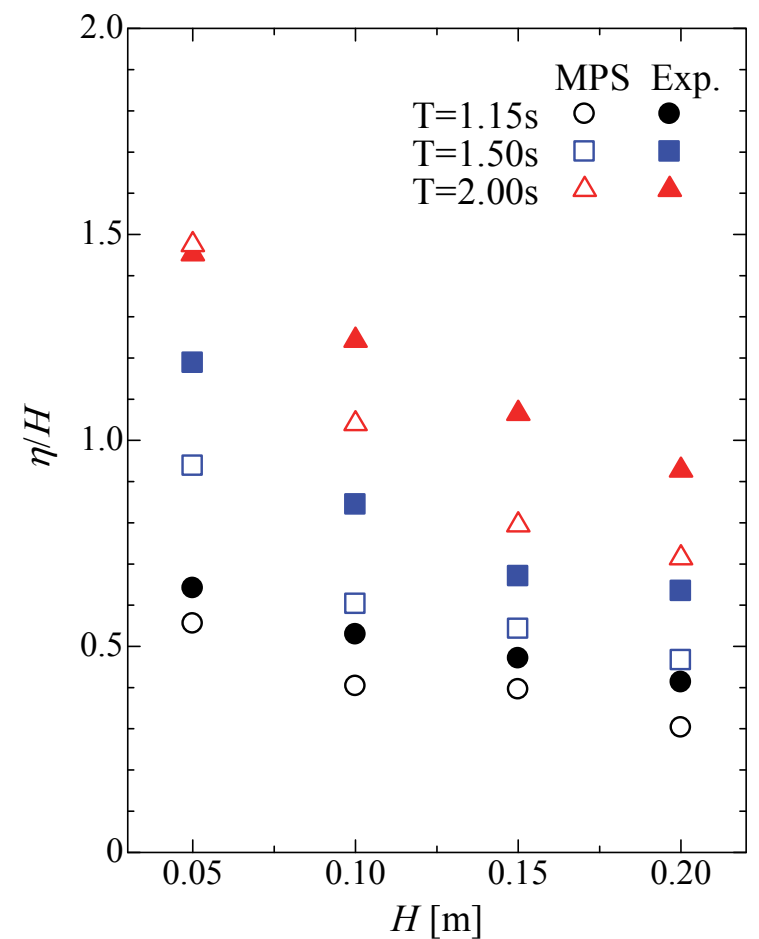

Figure 12 The comparison of the non-dimensional water elevations in the air chamber

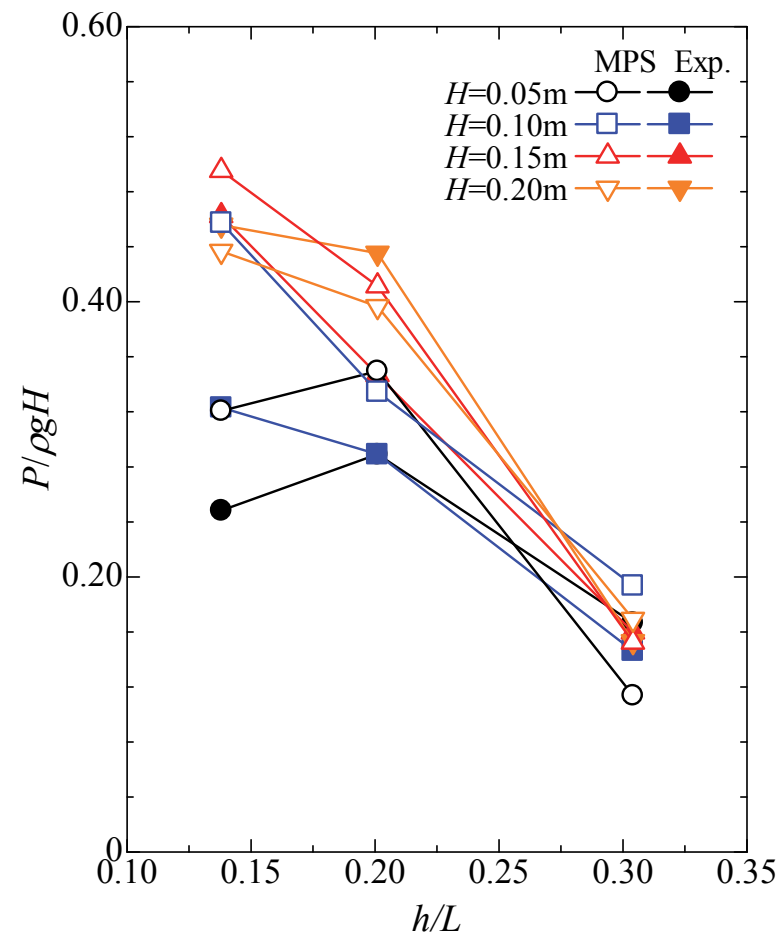

Figure 13 The comparison of

the non-dimensional positive pressures in the air chamber

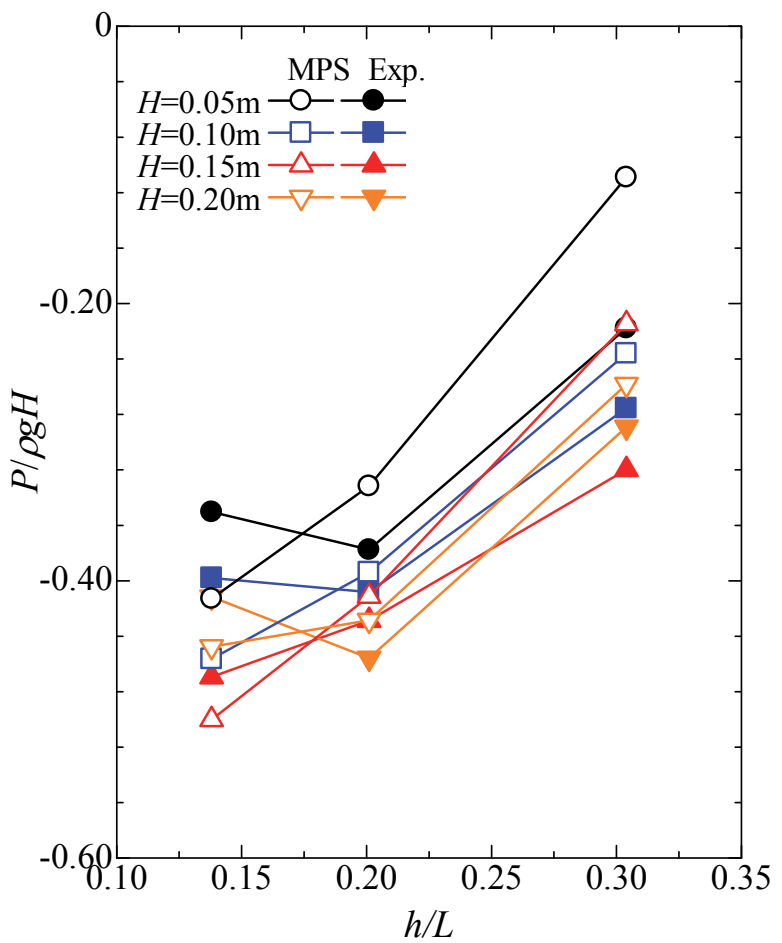

Figure 14 The comparison of the non-dimensional negative pressures in the air chamber

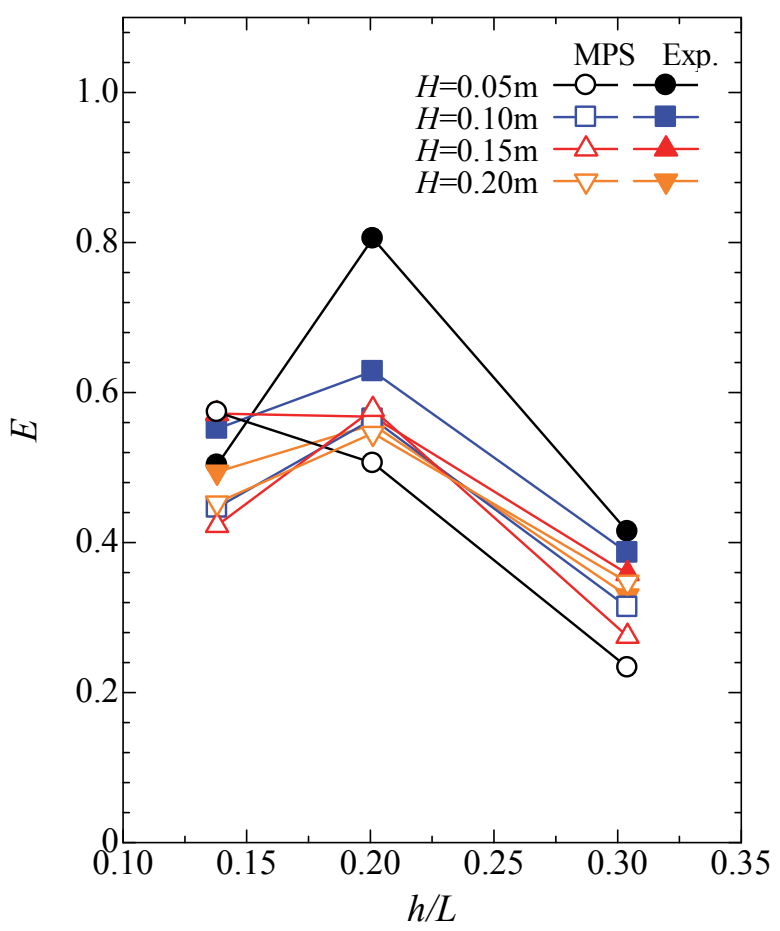

Figure 15 The comparison of the primary conversion efficiencies

\section{CONCLUSIONS}

The conclusions obtained in the present study are given as follows:

1. The MPS method was applied for the performance analysis of the OWC-WEC. In this study, the multiphase flow model, the improved source term of the pressure Poisson equation, the high accuracy gradient model, the divergence model and the atmospheric pressure model were applied to the MPS method for high accuracy OWC-WEC simulation.

2. It was found that this improved MPS program code can accurately calculate the inflow and outflow of the air in the air chamber. By adapting the nozzle boundary, it is possible to drastically reduce the number of particles required for simulation. 
3. In the previous MPS method, non-physical blank areas occur due to the judgement condition of the free boundary surface. This improved MPS method do improved the judgement condition of the free boundary surface of the air particles, and the non-physical blank areas do not occur. With this improvement, it is possible to calculate with high accuracy the air pressure in the air chamber.

4. Two dimensional numerical simulation was conducted by simulating the experimental results of the fixed type OWC-WEC. By comparison with the numerical result and the experimental results, it was found that the simulation results and the experimental results are broadly similar. However, when the wave height is low, the calculation accuracy becomes low due to the three-dimensional effects and the influence of the depth of the curtain wall. This MPS method cannot reproduce the thin curtain wall using relatively large size particles. In future work, it is necessary that those issues are solved and the water tank experiment for these simulation results is conducted.

In the future, it is necessary to consider a method to reproduce the thin curtain wall using relatively large size particles. Additionally, further verification of the calculation accuracy of the air particles is necessary. It is also necessary that the water tank experiment for these simulation results is conducted.

\section{ACKNOWLEGMENTS}

We are grateful to T. Nagase for assistance with the numerical simulations and the data organization. This research was made possible by the Grant in Aid for Scientific Research B of the Ministry of Education, Culture, Sports, Science and Technology.

\section{REFERENCES}

[1] Masuda, Y. and McCormick, ME., Experiences in Pneumatic Wave Energy Conversion in Japan, McCormick ME, Kim YC editors, Utilization of Ocean Waves - Wave to Energy Conversion, New York: ASCE, pp. 1-33, 1986.

[2] Washio, Y., Osawa, H., Ogata, T., Nakagawa, H., Okayama, S. and Nagata, Y., A Study on Characteristics of Generated Output of the Offshore Floating Type Wave Pert Device "Mighty Whale", Journal of the Society of Naval Architects of Japan, 190, pp. 395-405, 2001-12.

[3] The Queen's University of Belfast: Islay LIMPET Wave Power Plant Publishable Report, 2002.

[4] Masuda, M., Ikoma, T., Masuda, K. and Maeda, H., A Study on Predictions of Fully Nonlinear Motion of Aircushion Type Floating Structures Using 2D MPS Method, Proc. of OMAE'09, ASME, 57387, 2008.

[5] Koshizuka, S. and Oka, Y., Moving-Particle Semi-implicit Method for Fragmentation of Incompressible Fluid, Nucl. Sci. Eng., 123, pp. 421-434, 1996.

[6] Koshizuka, S., Nobe, A. and Oka, Y., Numerical Analysis of Breaking Waves Using the Moving Particle Semi-implicit Method, Int. J/. Numer. Meth. Fluids, 26, pp. 751-769, 1998.

[7] Masuda, K., Masuda, M., Minami, K and Ikoma, T., Masato OHNO and Fuminori NAKAMURA: Study on Development of the Floating Pier for Damage Protection Control of Ship in Tsunami, Proc. of OMAE'14, ASME, $24428,2014$.

[8] Masuda, M., Masuda, K., Ikoma, T. and Shoji, K., Behavior of the Pontoon Supported by Mooring Dolphin in Tsunami (First Report), Journal of OMAE, ASME, Vol.135, No. 2, pp. 021102-1-7, 2013.

[9] Koshizuka, S., Ikeda, H. and Oka, Y., Numerical Analysis of Fragmentation Mechanisms in Vapor Explosions, Nuclear Eng. and Des., Vol. 189, pp. 423-433, 1999-5.

[10] Arai, J., Numerical Analysis of the Free Surface Waves Around Wedge Models, Conference Proceedings, the Japan Society of Naval Architects and Ocean Engineers, JASNAOE, Vol. 12, pp. 445-448, 2011-5.

[11] Sueyoshi, M., Development of a Hybrid Technique of Particle Method and Boundary Element Method for Numerical Simulation of Water Waves, Reports of Research Institute for Applied Mechanics, Kyushu University, Vol. 133, pp.155-160, 2007-9.

[12] Hirata, N. and Anzai, K., Numerical Simulation of Shrinkage Formation of Pure Sn Casting Using Particle Method, Materials Transactions, Vol. 52, No. 10, pp. 1931-1938, 2011.

[13] Iribe, T. and Nakaza, E., A Precise Calculation Method of the Gradient Operator in Numerical Computation with the MPS, Journal of Japan Society of Civil Engineers, Ser. B2 (Coastal Engineering), JSCE, Vol. 66, No. 1, pp. 46-50, 2010.

[14] Tamai, K., Koshizuka, S. and Shibata, K., Development of High-Accuracy Models for Particle Interactions in the MPS Method, Proceedings of the Conference on Computational Engineering and Science, JSCES, Vol. 17, C-2-2, $2012-5$.

[15] Shibata, K., Murozono, K. and Kondo, M., Sakai, M. and Koshizuka, S., Numerical Modeling of Gas-phase Pressure, Negative Pressure and Curl Operator by the MPS Method, Proceedings of the Conference on Computational Engineering and Science, JSCES, Vol. 17, C-2-3, 2012-5.

[16] Shibata, K., Koshizuka, S., Sakai, M., Tanizawa, K. and Tsujimoto, M., Transparent Boundary Condition for Calculating Water Waves by a Particle Method, Journal of the Japan Society of Naval Architects and Ocean Engineers, JASNAOE, Vol. 11 , pp. 125-136, 2010.

[17] Ojima, R., Goda, Y. and Suzumura, S., Analysis of Efficiency of Pneumatic-type Wave Power Extractors Utilizing Caisson Breakwaters -A Study on Development of Wave Power 1st Report-, Report of the Port and Harbour Research Institute, Ministry of Transport, Vol. 22, No. 3, pp. 115-122, 1983-9.

[18] Kawaguchi, T., Absorbing Wave Making System with Wave Sensor and Velocity Control, Mitsui Zosen Technical Review, Mitsui E\&S, Vol. 128, pp. 20-24, 1986.

[19] Tanaka, S., Noto, H. and Takewaka, S., Study on Improvement of Oscillating Water Column Wave Power Generation, Journal of Japan Society of Civil Engineers, Ser. B3 (Ocean Engineering), Vol. 69, No. 2, pp. I_126-I_131, 2013. 